Nonlin. Processes Geophys., 25, 477-479, 2018

https://doi.org/10.5194/npg-25-477-2018

(C) Author(s) 2018. This work is distributed under

the Creative Commons Attribution 4.0 License.

\title{
Preface: Nonlinear waves and chaos
}

\author{
Gurbax S. Lakhina ${ }^{1}$, Bruce T. Tsurutani ${ }^{2}$, George J. Morales ${ }^{3}$, Annick Pouquet ${ }^{4}$, Masahiro Hoshino ${ }^{5}$, \\ Juan Alejandro Valdivia ${ }^{6}$, Yasuhito Narita ${ }^{7}$, and Roger Grimshaw ${ }^{8}$ \\ ${ }^{1}$ Indian Institute of Geomagnetism, New Panvel (W), Navi Mumbai, India \\ ${ }^{2}$ Jet Propulsion Laboratory, California Institute of Technology, Pasadena, CA, USA \\ ${ }^{3}$ Physics and Astronomy Department, University of California Los Angeles, Los Angeles, CA, USA \\ ${ }^{4}$ UCAR, Boulder, CO, USA \\ ${ }^{5}$ Department of Earth and Planetary Physics, University of Tokyo, Tokyo, Japan \\ ${ }^{6}$ Departamento de Fisica, Facultad de Ciencias, Universidad de Chile, Santiago, Chile \\ ${ }^{7}$ Austrian Academy of Sciences, Graz, Austria \\ ${ }^{8}$ Department of Mathematics, University College London, London, WC1E 6BT, UK
}

Correspondence: Gurbax S. Lakhina (gslakhina@gmail.com)

Received: 20 June 2018 - Published: 29 June 2018

Nonlinear waves and chaos play important roles in the acceleration and heating of charged particles and the generation of turbulence in astrophysical, space, and laboratory plasmas. A series of workshops on "Nonlinear Waves and Chaos" have been held to discuss this subject in Kyoto, Japan (1994), Cologne, Germany (1997), Carlsbad, USA (1999), Tromsø, Norway (2001), Mumbai, India (2003), Fukuoka, Japan (2006), Beaulieu-surMer, France (2008), and La Jolla, USA (2010, 2013). The 10th International Nonlinear Waves and Chaos Workshop 2017 (NWCW17) was held in San Diego, California, from 20 to 24 March 2017.

This workshop was unique because it brought together 34 experts, including space, laboratory, and fusion plasma physicists, astrophysicists, applied mathematicians, atmospheric and oceanic scientists, and even a heart specialist, from various countries. Since nonlinear waves, chaos, dispersion, and dissipation occur in many fields of natural and biological sciences, it is therefore desirable to compare theoretical approaches and observational techniques from various disciplines. This workshop provided an opportunity to report on the latest results from theory, simulation, and data analysis dealing with the nonlinear processes and complex system dynamics in space, laboratory, and astrophysical plasmas as well as rogue ocean waves and cardiac arrhythmia.

This special issue of Nonlinear Processes in Geophysics $(N P G)$ consists of papers drawn from the talks given at the NWCW 2017 as well as papers that were submitted in response to the call for papers for this special issue. All papers had to undergo the usual $N P G$ review process before they were finally accepted for this special issue.

The papers in this special issue cover a broad range of topics related to nonlinear waves and chaos. These include intermittent turbulence in the heliosheath and magnetosheath plasmas, the evolution of fractality in magnetized plasmas, a lifetime estimate for solar wind plasma turbulence, the statistical origin of kappa distributions in space plasmas, the application of a dynamical system approach to study extremes of northern hemispheric climate fields over the past 60 years, an application of wavelet decomposition and reconstruction techniques to find correlations between Earth's auroral electrojet (AE) index and ACE solar wind magnetic data for predicting geomagnetic activity, and estimates of satellite drag effects due to uplifted oxygen neutral atoms during super magnetic storms. A brief summary of the special issue papers is given below.

Livadiotis (2018) presents results on the statistical origin of kappa distributions, which describe velocities and energies of electrons and ions in space plasmas, in terms of nonextensive statistical mechanics. The kappa distribution can be derived by maximizing the $q$-entropy of Tsallis under the constraints of a canonical ensemble. However, the physical origin of this entropic formulation is not known. This paper shows that the $q$-entropy can be derived by adapting the additivity of energy and entropy. 
Guarnieri et al. (2018) discuss the possibility of predicting geomagnetic activity based on the correlations between the AE index and the solar wind magnetic field data series based on wavelet decomposition and reconstruction techniques. The filtered interplanetary magnetic field (IMF) data are fed into a model to calculate a time series, which is called $\mathrm{AE}^{*}$. Empirical removal of the high-frequency, shortwavelength Alfvénic component in the IMF by wavelet decomposition is shown to dramatically improve the correlation between $\mathrm{AE}^{*}$ and the actual measured $\mathrm{AE}$ index. They propose that this $\mathrm{AE}^{*}$ can be used as the main input for a model to forecast the acceleration of relativistic electrons in the Earth's outer radiation belts, which are delayed by $\sim 1$ to 2 days from intense $\mathrm{AE}$ events.

Macek et al. (2018) show that solar wind turbulence over the entire heliosphere is intermittent. By analyzing the fluctuations of plasma and magnetic field parameters observed by THEMIS, they find that plasma turbulence behind quasiperpendicular shocks is more intermittent with larger kurtosis than that behind quasi-parallel shocks. Further, behind the bow shock, turbulence is often more intermittent at higher Alfvénic Mach numbers than at lower values. The level of intermittency for the outgoing and incoming waves seems to be similar. This is consistent with the equipartition of energy between these oppositely propagating Alfvén waves.

Narita and Vörös (2017) propose a novel method, based on the applicability of the Breit-Wigner spectrum model with a non-Gaussian frequency broadening to the multi-spacecraft Cluster data, to estimate the decay rate of turbulent fluctuations in the solar wind experimentally. Their estimate for the spatial scale of decay rate is about $1000 \mathrm{~km}$ (about 10 times larger than the ion inertial length).

Lakhina and Tsurutani (2017) report on the satellite drag effect of uplifted oxygen neutrals during super magnetic storms, like the 1-2 September 1859 Carrington superstorm. During intense magnetic storms, prompt penetration electric fields, through $\mathrm{E} \times \mathrm{B}$ forces near the magnetic equator, uplift the dayside ionosphere. Ion-neutral drag forces between the upward moving $\mathrm{O}^{+}$(oxygen ions) and oxygen neutrals can elevate the oxygen atoms to higher altitudes. Based on linear analysis, they estimate that with a prompt penetrating electric field of $\sim 20 \mathrm{mV} \mathrm{m}^{-1}$ applied for $20 \mathrm{~min}$, the $\mathrm{O}$ atoms and $\mathrm{O}^{+}$ions are uplifted to $850 \mathrm{~km}$, where they produce about 40 times greater satellite drag per unit mass than normal. It is concluded that during Carrington-type magnetic storms, the oxygen neutral densities at low-Earth-orbiting satellite altitudes may be sufficiently high to present severe satellite drag.

Faranda et al. (2017) report results on the application of a dynamical system approach to explore extremes of northern hemispheric climate fields over the past 60 years. Under this approach the atmospheric motions are chaotic but not random: their trajectories stay close to a high-dimensional object called an attractor. The authors analyze the data on sea-level pressure, $2 \mathrm{~m}$ temperature (air temperature observations taken $2 \mathrm{~m}$ above ground), and precipitation frequency for the Northern Hemisphere and estimate two instantaneous dynamical properties: local dimension and persistence. Their results show that (i) despite the high dimensionality of atmospheric dynamics, the northern hemispheric sea-level pressure and temperature fields can on average be described by roughly 20 degrees of freedom; (ii) the precipitation field has a higher dimensionality; and (iii) the seasonal forcing modulates the variability of the dynamical indicators and affects the occurrence of phase-space extremes.

Muñoz et al. (2018) report results on the temporal evolution of fractality for geomagnetic activity. They calculate fractal dimensions from disturbance storm time (Dst) data and from a magnetohydrodynamic (MHD) shell model for a turbulent magnetized plasma. In certain regions of viscosity and resistivity value ranges, the shell model is able to reproduce the relationship between the fractal dimension and the occurrence of dissipative events. They apply these ideas to the study of the solar wind magnetic field data time series during magnetic cloud events. Their results suggest that the fractal dimension is able to characterize the complexity of the magnetic cloud structure. This model can be used to study geomagnetic activity under solar wind forcing.

Acknowledgements. We sincerely thank all the referees who gave their time to carefully review the above papers and give valuable suggestions/comments which led to their improvement. We hope that the readers of $N P G$ will enjoy this special issue. A portion of this work was done at the NASA Jet Propulsion Laboratory, California Institute of Technology, Pasadena, CA, USA. Gurbax S. Lakhina thanks the Indian National Science Academy, New Delhi, India, for support under the INSA Honorary Scientist Scheme.

\section{References}

Faranda, D., Messori, G., Alvarez-Castro, M. C., and Yiou, P.: Dynamical properties and extremes of Northern Hemisphere climate fields over the past 60 years, Nonlin. Processes Geophys., 24, 713-725, https://doi.org/10.5194/npg-24-713-2017, 2017.

Guarnieri, F. L., Tsurutani, B. T., Vieira, L. E. A., Hajra, R., Echer, E., Mannucci, A. J., and Gonzalez, W. D.: A correlation study regarding the AE index and ACE solar wind data for Alfvénic intervals using wavelet decomposition and reconstruction, Nonlin. Processes Geophys., 25, 67-76, https://doi.org/10.5194/npg-2567-2018, 2018.

Lakhina, G. S. and Tsurutani, B. T.: Satellite drag effects due to uplifted oxygen neutrals during super magnetic storms, Nonlin. Processes Geophys., 24, 745-750, https://doi.org/10.5194/npg24-745-2017, 2017.

Livadiotis, G.: Derivation of the entropic formula for the statistical mechanics of space plasmas, Nonlin. Processes Geophys., 25, 77-88, https://doi.org/10.5194/npg-25-77-2018, 2018.

Macek, W. M., Wawrzaszek, A., and Kucharuk, B.: Intermittent turbulence in the heliosheath and the magnetosheath plasmas based on Voyager and THEMIS data, Nonlin. Processes Geophys., 25, 39-54, https://doi.org/10.5194/npg-25-39-2018, 2018. 
Muñoz, V., Domínguez, M., Valdivia, J. A., Good, S., Nigro, G., and Carbone, V.: Evolution of fractality in space plasmas of interest to geomagnetic activity, Nonlin. Processes Geophys., 25, 207216, https://doi.org/10.5194/npg-25-207-2018, 2018.
Narita, Y. and Vörös, Z.: Lifetime estimate for plasma turbulence, Nonlin. Processes Geophys., 24, 673-679, https://doi.org/10.5194/npg-24-673-2017, 2017. 